\title{
Gelatinase-Associated Phenotypes and Genotypes Among Clinical Isolates of Enterococcus faecalis in Poland
}

\author{
JANUSZ STRZELECKI, WALERIA HRYNIEWICZ and EWA SADOWY*
}

National Medicines Institute, Department of Molecular Microbiology, Warsaw, Poland

Received 11 February 2011, revised 10 August 2011, accepted 16 August 2011

\author{
Abstract
}

\begin{abstract}
Enterococcus faecalis is an important nosocomial pathogen causing serious invasive infections. One of the virulence factors of this pathogen, gelatinase GelE, is a protease whose gene expression is regulated by the Fsr quorum sensing system. In this study, we used a wellcharacterized collection of 153 clinical E. faecalis isolates to investigate the distribution of genes involved in gelatinase expression. Although 140 isolates (91\% of the group) harbored the gelE gene, only 81 isolates (53\%) produced active gelatinase. The gelatinase-negative phenotype was found in several unrelated clones, and appeared to be caused by various genetic events. Isolates of the hospital-adapted clonal complex 2 (CC2) and of CC40 were uniformly gelatinase-positive, while all the CC87 isolates contained the $23.9 \mathrm{~kb}$ deletion encompassing most of the $f_{s} r$ locus and were gelatinase-negative. No significant differences among isolates of different clinical origin and gelatinase activity or presence of the $f s r$ genes were found with the exception of isolates from cerebrospinal fluid, which were more often gelatinase-positive than colonizing isolates.
\end{abstract}

Key words: E. faecalis, clone, fsr, gelatinase

\section{Introduction}

Enterococci represent important nosocomial pathogens worldwide (Werner et al., 2008), and the major clinically relevant species, Enterococcus faecalis causes serious invasive infections, such as endocarditis and bacteremia as well as urinary tract and surgical site infections (Arias et al., 2010). The treatment is complicated by increasing resistance of the species to several antimicrobial agents (Arias et al., 2010), observed also in Poland (Rudy et al., 2005; Kawalec et al., 2007; Piekarska et al., 2008). Several enterococcal virulence factors have been identified and studied extensively (Fisher and Phillips, 2009; Hendrickx et al., 2009). One of them, gelatinase (GelE) of E. faecalis is an extracellular zinc metalloendopeptidase (Makinen et al., 1989), whose gene ( $g e l E)$ is co-transcribed with the located directly downstream $\operatorname{sprE}$ gene encoding serine protease, SprE (Qin et al., 2000; Qin et al., 2001). These proteases provide peptide nutrients to bacteria, as well as their activity causes direct and indirect damage to host tissues (Gilmore, 2002). A number of studies demonstrated that GelE enhances biofilm production (Hancock and Perego, 2004; Kristich et al., 2004; Mohamed etal., 2004), whereas SprE has an oppo- site effect (Thomas et al., 2008). The interplay of GelE and SprE regulates autolysis of E. faecalis cells and the subsequent release of extracellular DNA (eDNA), a critical structural component of biofilm produced by these bacteria (Guiton et al. 2010; Thomas et al., 2008). GelE appears also important for cleavage of pheromones responsible for induction of conjugal plasmid transfer and degradation of misfolded bacterial proteins (Waters et al., 2003).

The expression of $g e l E$ and $s p r E$ is regulated by the fsr locus (Nakayama et al., 2001), a homologue of agr of Staphylococcus aureus (Balaban and Novick, 1995; Qin et al., 2000). FsrA and FrrC constitute a two-component signal transduction system (TCS), in which FsrA acts as response regulator and $\mathrm{FsrC}$ as signal transducer, responding to an increasing extracellular concentration of an 11-amino-acid cyclic peptide, so-called gelatinase biosynthesis-activating pheromone (GBAP). Activated FsrC phosphorylates FsrA which induces transcription of gelE and sprE, and regulates several other genes, involved in pathogenesis and metabolic pathways (Qin et al., 2000). The GBAP coding sequence was identified within the $f s r D$ gene, which is contained in frame in the 3'terminal part of $f_{s} r B$, but independently translated (Nakayama et al., 2006). The $f s r B$ product is probably

\footnotetext{
* Corresponding author: E. Sadowy, Department of Molecular Microbiology, National Medicines Institute; Chełmska 30/34, 00-725 Warsaw, Poland; phone: +48 2285143 88, fax: +48 2284129 49; e-mail: ewasadowy@cls.edu.pl
} 
involved in the FsrD processing and transport outside the cell (Nakayama et al., 2001; Nakayama et al., 2006). In some isolates, a naturally occurring $23.9-\mathrm{kb}$ chromosomal deletion, encompassing $f_{s} r A, f s r B$ and a part of $f s r C$, resulting in the gelatinase-negative phenotype was identified (Nakayama et al., 2002). A number of studies using animal models have shown the contribution of GelE, SprE and Fsr to virulence of E. faecalis. In a mouse peritonitis model, the gelatinase mutant was attenuated; similarly, sprE, fsr $A$ and $f s r B$ mutants also showed delayed time of killing compared to the wild-type strain (Qin et al., 2000). The virulence of $f s r$ mutants was lower than that of a deletion mutant of gelE and an insertion mutant in sprE (Sifri et al., 2002). Studies using a rabbit endophthalmitis model, an exquisitely sensitive infection system for investigating quorum sensing mechanisms, gave similar results, suggesting possible pleiotropic contribution of the $f s r$ locus to the virulence (Engelbert et al., 2004).

Recently, multilocus sequence typing (MLST) has become a method of choice for typing of E. faecalis. In the study that introduced the current typing scheme based on seven house-keeping genes, MLST was used for analysis of 110 E. faecalis isolates from different sources and geographic locations (Ruiz-Garbajosa etal., 2006). Among four major clonal complexes (CCs) identified, CC2 and CC9 were considered to be hospital-adapted and spread globally. Another study, performed on the collection of E. faecalis from hospitals in Poland demonstrated the presence of additional three major endemic clones apart from CC2 and CC9 in this country (Kawalec et al., 2007). We used this wellcharacterized collection to investigate the distribution of gelE, sprE and fsr genes, and to compare these data with earlier MLST results.

\section{Experimental}

\section{Material and Methods}

Bacterial isolates. A hundred and fifty-three isolates collected by the National Medicines Institute in Warsaw from 42 medical centers in 28 Polish cities during 1996-2005 were used in this study. These isolates were obtained from various sources: 54 isolates were from invasive infections (blood, peritoneal, cerebrospinal and pleural fluids), 52 isolates were from non-invasive infections (wound, urine, eye), 40 isolates were from carriage (rectum, feces, skin, throat, sputum, vagina, sperm, ear), six isolates were from colonization of catheters and drains, and one isolate was from the hospital environment (Kawalec et al., 2007).

Determination of gelatinase activity. The phenotypic assay of gelatinase activity was performed accord- ing to the modified method described by Su et al. ( $\mathrm{Su}$ et al., 1991). A $10 \mu \mathrm{l}$ drop of an overnight culture in Bacto Todd-Hewitt Broth (Becton Dickinson, Sparks, $\mathrm{MD}$ ) was spotted on a plate with medium containing $3 \%$ gelatin (Oxoid, Hampshire, England); 0.5\% peptone (Becton Dickinson); 0.3\% yeast extract (Becton Dickinson), 1.5\% agar (Becton Dickinson); pH 7; after $48 \mathrm{~h}$ incubation at $37^{\circ} \mathrm{C}$ in aerobic conditions, the plate surface was covered with a saturated solution of ammonium sulphate (Sigma-Aldrich Chemie, Steincheim, Germany). Presence of a transparent zone around colonies indicated gelatinase activity.

MLST and detection of gelE, sprE, fsrA and $23.9 \mathrm{~kb}$ deletion by PCR. DNA isolation was performed with Genomic Mini kit (A\&A Biotechnology, Gdańsk, Poland) according to the manufacturer's instructions. MLST was performed using the standard procedure (Ruiz-Garbajosa et al., 2006) and the database (http:// www.mlst.net/) to assign alleles and sequence types (STs). Sequences were assembled using the SeqMan program in DNASTAR software package (Lasergene, Madison, WI) and results were analyzed with the eBURST software (Feil et al., 2004) with members of a CC sharing six out of the seven MLST loci (singlelocus variants, SLVs). The presence of gelE, sprE and $f_{s} r A$ was determined by PCR with primers described by Lopes et al. (Lopes et al., 2006). The amplification was performed in the following conditions: $2 \mathrm{~min}$ at $94^{\circ} \mathrm{C}$; $30 \mathrm{~s}$ at $94^{\circ} \mathrm{C}, 30 \mathrm{~s}$ at $55^{\circ} \mathrm{C}, 1 \mathrm{~min}$ at $72^{\circ} \mathrm{C}$ for 35 cycles; $7 \mathrm{~min}$ at $72^{\circ} \mathrm{C}$. DNA of E. faecalis V583 was used as a positive control. For the PCR detection of the $23.9 \mathrm{~kb}$ deletion in the $f s r$ locus, primers proposed by Roberts et al. (Roberts et al., 2004) were used and the elongation time was extended to $2 \mathrm{~min}$. PCR products were analyzed by $1.5 \%$ agarose gels electrophoresis (SeaKem LE Agarose, Lonza, Rockland, MN), stained with ethidium bromide (Sigma-Aldrich Chemie).

Dot-blot hybridization assay. For dot-blot hybridization, $50 \mathrm{ng}$ of chromosomal DNA was denaturated with $0.4 \mathrm{M} \mathrm{NaOH}$ and $10 \mathrm{mM}$ EDTA solution for $5 \mathrm{~min}$, and vacuum blotted to a Hybond ${ }^{+}$nylon membrane (GE Healthcare, Buckinghamshire, UK) in a Bio-Dot Microfiltration Apparatus (Bio-Rad, Hercules, CA). DNA was fixed to the membrane by a $0.4 \mathrm{M} \mathrm{NaOH}$ wash, followed by neutralization with $2 \times$ SSC and exposure to UV radiation for $2 \mathrm{~min}$ as recommended by the vacuum-blotter manufacturer. Gene-specific probes for gelE, sprE, and $f s r$ genes were generated by PCR using primers described by Lopes et al. (Lopes et al., 2006). Probe labeling and signal detection were carried out according to the ECL Direct Nucleic Labeling and Detection System (GE Healthcare). Membranes were hybridized overnight at $42^{\circ} \mathrm{C}$ with $100 \mathrm{ng}$ of a probe, washed, incubated with the detection reagents, and exposed on a X-ray film (X-ray Retina Fotochemische 
Werke GmbH, Berlin, Germany), which was developed and fixed using Kodak processing chemicals (SigmaAldrich Chemie).

Statistical analysis. The chi-squared test was used to evaluate the significance of differences in isolate distributions.

\section{Results}

The activity of gelatinase was initially characterized in the previous study (Kawalec et al., 2007) and these data were verified in the current work for reproducibility and a possible loss of phenotype during storage of isolates. These results were entirely congruent with the previous data. Among 153 E. faecalis isolates, the gelatinase-positive phenotype $\left(\mathrm{Gel}^{+}\right)$was found for 81 isolates (53\%). In all the $\mathrm{Gel}^{+}$isolates, the gelE, sprE and $f s r A$ genes were detected by both PCR and hybridization, and the presence of $f_{s r} B$ and $f_{s r} C$ was confirmed by hybridization for a few randomly chosen representatives of this group (the gelE$^{+}, s p r E^{+}$and $f s r^{+}$genotype, Table I). In the group of 72 gelatinase-negative $\left(\mathrm{Gel}^{-}\right)$ isolates, 39 showed the presence of the $23.9-\mathrm{kb}$ deletion by PCR ( $g e l E^{+}, s p r E^{+}$and $\left.\Delta f s r\right)$. As expected, a few checked representatives of this group were negative for $f_{s} r B$ but positive for $f s r C$ in hybridization. All the remaining 33 isolates were additionally tested for the presence of $f_{s r} B$ and $f s r C$ by hybridization and showed three different genotypes (Table I). Nine isolates had a complete set of the gelE, sprE and fsr genes; 11 isolates possessed gelE and $s p r E$ but lacked all $f s r$ genes and did not show the 23.9-kb deletion by PCR; thirteen isolates lacked all the investigated genes.

The frequency of gelatinase production and the prevalence of $f s r$ genes varied depending on the iso- lation site and these differences were statistically significant $(p=0.009$ and $p=0.008$, respectively, for the whole group). The gelatinase production was common among isolates from the cerebrospinal fluid $(75 \%$, i.e. $6 \mathrm{Gel}^{+}$isolates out of 8$)$, urine $(71 \%, 17 / 24)$ and vagina $(70 \%, 14 / 20)$, less frequent for wound and blood isolates $(50 \%, 14 / 28$ and $44 \%, 15 / 34$, respectively) and the rarest among isolates from rectum and feces $(27 \%$, $3 / 11)$ and peritonitis $(10 \%, 1 / 10)$.

Gelatinase phenotypes and genotypes were next analyzed in the context of molecular typing data. The previous MLST results (Kawalec et al., 2007) were corrected for four STs, i.e. STs 131, 6, 137 and 121 were changed to STs 378 (a new ST), 88, 8 and 220, respectively. In summary, the investigated group of 153 isolates contained 52 STs, grouped into five CCs $(21,40$, 87,88 and 141) and 42 unrelated STs (singletons) by the eBURST analysis. One of the previously defined major epidemic clones, CC2 (the so-called clone A, represented here by 10 isolates of ST6) uniformly produced gelatinase (Table I). All the representatives of the other epidemic clone, CC9 (clone J, 6 isolates of ST9) possessed the full set of analyzed genes; however, one isolate of this ST lost the ability to produce gelatinase. Of the Polish endemic clones, isolates belonging to the previously described clone F (24 isolates of CC87) were all gelatinase-negative due to the presence of the 23.9-kb deletion. In the clone $\mathrm{C}$, consisting of two subclones unrelated in MLST, i.e. ST16 and CC40, all CC40 isolates were gelatinase producers, while among representatives of ST16, two out of six isolates lacked the gelE, sprE and $f_{s r}$ genes. Finally, clone I (CC21, 19 isolates) included six $\mathrm{Gel}^{+}$and thirteen $\mathrm{Gel}^{-}$isolates; of the latter, ten possessed gelE and sprE but lacked the $f s r$ genes, two isolates lacked the gelE, sprE and $f s r$ genes and one possessed the full set of analyzed genes.

Table I

Fenotypes and genotypes of $E$. faecalis analyzed in this study in relation to MLST results

\begin{tabular}{|c|c|c|c|c|c|c|c|c|c|}
\hline $\begin{array}{l}\text { Gelatinase } \\
\text { phenotype }\end{array}$ & gelE & $s p r E$ & $f s r A$ & $f s r B$ & $f_{s r C}$ & $\begin{array}{l}23.9 \mathrm{~kb} \\
\text { deletion }\end{array}$ & $\begin{array}{c}\begin{array}{c}\text { Number } \\
\text { of isolates }\end{array} \\
\end{array}$ & \begin{tabular}{|c|} 
Number of \\
invasive isolates
\end{tabular} & MLST \\
\hline+ & + & + & + & $+^{\mathrm{a}}$ & $+^{\mathrm{a}}$ & nd & 81 & 23 & $\begin{array}{l}\text { CCs: } \underline{21}(6), 40(26), 141(2) \\
\text { STs: } 6(10), \underline{9}(5), \underline{16}(4), 47(4), 59(2), 72(2), 81 \text {, } \\
82,86,93,102,119,122,123,124,125,126,127, \\
128,130,134,136,138,139,142,143\end{array}$ \\
\hline- & + & + & - & $-^{\mathrm{a}}$ & $+^{\mathrm{a}}$ & + & 39 & 13 & $\begin{array}{l}\text { CCs: } 87 \text { (24) } \\
\text { STs: } 8 \text { (3), } \underline{25}(2), 26 \text { (2), } 30 \text { (6), } 41 \text { (2) }\end{array}$ \\
\hline- & + & + & + & + & + & - & 9 & 6 & $\begin{array}{l}\text { CCs: } \underline{21}, 88(5) \\
\text { STs: } \underline{9}, \underline{25}, 120\end{array}$ \\
\hline- & + & + & - & - & - & - & 11 & 5 & $\begin{array}{l}\text { CCs: } \underline{21}(10) \\
\text { ST: } 140\end{array}$ \\
\hline- & - & - & - & - & - & - & 13 & 7 & $\begin{array}{l}\text { CCs: } \underline{21}(2) \\
\text { STs: } \underline{16}(2), 23,55(2), 74,84,85,133,135,378\end{array}$ \\
\hline
\end{tabular}

a detection by dot-blot performed for representatives; nd, not determined.

The numbers of isolates, if higher than one, are given in the brackets; CCs and STs present in more than one group are underlined. 
The majority of sporadic isolates produced gelatinase and had the $g e l E^{+}, s p r E^{+}$and $f s r^{+}$genotype.

\section{Discussion}

The isolates used in this study were typed previously by pulsed-field electrophoresis (PFGE) and MLST, and a number of major clones were described (Kawalec et al., 2007). The aim of the present research was to investigate the distribution of the gelE and $s p r E$ genes, and genes involved in the regulation of gelatinase expression in this well-characterized collection.

The gelatinase coding gene was present in $91 \%$ of isolates tested (140/153), whereas the gelatinase activity was found in only $53 \%(81 / 153)$ of them. In other studies, involving large groups of isolates from various clinical materials, it was shown that $56 \%$ and $59 \%$ of isolates produced gelatinase, while $88 \%$ and $92 \%$ were gelE-positive, respectively (Roberts et al., 2004; Mohamed and Murray, 2005). Among 31 endodontic E. faecalis, gelE was detected in all tested isolates, whereas the gelatinase activity was found in $74 \%$ of them (Sedgley et al., 2005). Certain studies, however, showed a lower prevalence of $\mathrm{gelE}^{+}$and $\mathrm{GelE}^{+}$isolates, accounting for $45-74 \%$ (Bittencourt de Marques and Suzart, 2004; Piekarska and Jagielski, 2007) and 34\% of analyzed collections of clinical isolates (Piekarska and Jagielski, 2007), respectively. The most common cause for a loss of gelatinase production is the $23.9 \mathrm{~kb}$ deletion encompassing most of the $f s r$ locus which was present e.g. in 26 of $33 \mathrm{Gel}^{-}$isolates from patients with urinary tract infections (Nakayama et al., 2002). Another likely cause for the gelatinase-negative phenotype is a point mutation in the gelE gene (Nakayama et al., 2002) or loss of the gene as observed e.g. for the genome of the probiotic strain Symbioflor (Domann et al., 2007).

In our study, the frequency of gelatinase activity and the presence of the $f s r$ genes varied depending on the site of isolation, being the highest for the isolates from the cerebrospinal fluid. The observation is consistent with the significantly increased prevalence of the $f_{s} r$ locus found for endocarditis isolates compared to fecal isolates (Pillai et al., 2002) as well as the fact that GelE plays an important role during infection in a rabbit endocarditis model (Thurlow et al., 2010) which altogether might indicate the importance of GelE and Fsr TCS for the most severe forms invasive enterococcal infections. Moreover, gelatinase production was also more often associated with hospital strains of $E$. faecalis than with strains carried in the community (Coque et al., 1995). The same study, however, did not show differences in gelatinase production among enterococci from different forms of hospital infections.
Comparison of the distribution of gelatinase activity and presence of the $f s r$ genes with the MLST results showed that these features are typical for hospitaladapted, globally spread CCs, such as CC2 and CC9, as well as for some Polish endemic clones, such as CC40 and most of ST16, while CC87 uniformly showed the presence of the $23.9-\mathrm{kb}$ deletion that excluded the production of gelatinase and activation of other genes dependent from Fsr TCS. We found the deletion also in some other unrelated singleton STs, likely due to a horizontal transfer of the corresponding region of the chromosome (Galloway-Pena et al., 2011). Another endemic clone, CC21 was the most diversified group, containing both $\mathrm{Gel}^{+}$and $\mathrm{Gel}^{-}$isolates, the latter showing various genotypes. In general, CC21 is a heterogeneous, not strictly hospital-adapted group, which encompasses also the isolates of animal and environmental origins (Ruiz-Garbajosa et al., 2006; Kawalec et al., 2007).

Although gelatinase is not essential for E. faecalis, it seems to play an important role, both in pathogenesis and in basic metabolic processes of the bacterium. This poses a question, why the $\mathrm{Gel}^{-}$phenotype has emerged in several unrelated clones due to various genetic events, such as deletion of the $f s r$ genes, horizontal transfer of the deletion-harboring region or presumable inactivation of the gelE gene. These observations suggest some evolutionary benefits associated with the loss of gelatinase production due to e.g. a significant cost of this process. It is also hypothesized that, as GelE and SprE are involved in the eDNA release necessary for biofilm formation, $\mathrm{Gel}^{-}$clones may be considered as "quorum sensing cheaters", that do not respond to the GBAP signal and do not contribute for the rest of the population but rather take advantage of it (Thomas et al., 2008; Thomas et al., 2009).

In conclusion, we used phenotypic and molecular methods for investigation of distribution of gelatinase and the $f_{s r}$ locus in a well-characterized collection of clinical isolates of E. faecalis and showed association of particular phenotypes and genotypes with enterococcal clones. The loss of gelatinase production appeared independently more than once in E. faecalis, however, certain hospital-adapted clones maintain this ability.

\section{Acknowledgements}

This publication made use of the Enterococcus faecalis MLST website (http://efaecalis.mlst.net/) hosted at Imperial College of the University of Oxford and funded by the Wellcome Trust. This work was supported by the ACE grant from the European Union VI Framework Programme under contract LSHE-CT-2007-037410 together with a complementary founding from the Ministry of Science and Higher Education (decision 937/6. PR UE/2009/7) and by MIKROBANK funding from the Ministry of Science and Higher Education, Poland. 


\section{Literature}

Arias C.A., G.A. Contreras and B.E. Murray. 2010. Management of multi-drug resistant enterococcal infections. Clin. Microbiol. Infect. 16: 555-562.

Balaban N. and R.P. Novick. 1995. Autocrine regulation of toxin synthesis by Staphylococcus aureus. Proc. Natl. Acad. Sci. USA 92: 1619-1623.

Bittencourt de Marques E. and S. Suzart. 2004. Occurrence of virulence-associated genes in clinical Enterococcus faecalis strains isolated in Londrina, Brazil. J. Med. Microbiol. 53: 1069-1073.

Coque T.M., J.E. Patterson, J.M. Steckelberg and B.E. Murray. 1995. Incidence of hemolysin, gelatinase, and aggregation substance among enterococci isolated from patients with endocarditis and other infections and from feces of hospitalized and communitybased persons. J. Infect. Dis. 171: 1223-1229.

Domann E., T. Hain, R. Ghai, A. Billion, C. Kuenne, K. Zimmermann and T. Chakraborty. 2007. Comparative genomic analysis for the presence of potential enterococcal virulence factors in the probiotic Enterococcus faecalis strain Symbioflor 1. Int. J. Med. Microbiol. 297: 533-539.

Engelbert M., E. Mylonakis, F.M. Ausubel, S.B. Calderwood and M.S. Gilmore. 2004. Contribution of gelatinase, serine protease, and $f s r$ to the pathogenesis of Enterococcus faecalis endophthalmitis. Infect. Immun. 72: 3628-3633.

Feil E.J., B.C. Li, D.M. Aanensen, W.P. Hanage and B.G. Spratt. 2004. eBURST: inferring patterns of evolutionary descent among clusters of related bacterial genotypes from multilocus sequence typing data. J. Bacteriol. 186: 1518-1530.

Fisher K. and C. Phillips. 2009. The ecology, epidemiology and virulence of Enterococcus. Microbiology. 155: 1749-1757.

Galloway-Pena J.R., A. Bourgogne, X. Qin and B.E. Murray. 2011. Diversity of the fsr-gelE region of Enterococcus faecalis but conservation in strains with partial deletion of the fsr operon. Appl. Environ. Microbiol. 77: 442-451.

Gilmore M.S., P.S. Coburn, S.R. Nallapareddy and B.E. Murray. 2002. Enterococcal virulence. pp. 301-354. In: Gimore M.S. (ed.). The Enterococci: Pathogenesis, Molecular Biology and Antibiotic Resistance. ASM Press, Washington, D.C.

Guiton P.S., C.S. Hung, L.E. Hancock, M.G. Caparon and S.J. Hultgren. 2010. Enterococcal biofilm formation and virulence in an optimized murine model of foreign body-associated urinary tract infections. Infect. Immun. 78: 4166-4175.

Hancock L.E. and M. Perego. 2004. The Enterococcus faecalis $f_{s r}$ two-component system controls biofilm development through production of gelatinase. J. Bacteriol. 186: 5629-5639.

Hendrickx A.P., R.J. Willems, M.J. Bonten and W. van Schaik. 2009. LPxTG surface proteins of enterococci. Trends Microbiol. 17: 423-430.

Kawalec M., Z. Pietras, E. Danilowicz, A. Jakubczak, M. Gniadkowski, W. Hryniewicz and R.J. Willems. 2007. Clonal structure of Enterococcus faecalis isolated from Polish hospitals: characterization of epidemic clones. J. Clin. Microbiol. 45: 147-153.

Kristich C.J., Y.H. Li, D.G. Cvitkovitch and G.M. Dunny. 2004. Esp-independent biofilm formation by Enterococcus faecalis. J. Bacteriol. 186: 154-163.

Lopes M. de F., A.P. Simoes, R. Tenreiro, J.J. Marques and M.T. Crespo. 2006. Activity and expression of a virulence factor, gelatinase, in dairy enterococci. Int. J. Food Microbiol. 112: 208-214. Makinen P.L., D.B. Clewell, F. An and K.K. Makinen. 1989. Purification and substrate specificity of a strongly hydrophobic extracellular metalloendopeptidase ("gelatinase") from Streptococcus faecalis (strain 0G1-10). J. Biol. Chem. 264: 3325-3334.
Mohamed J.A., W. Huang, S.R. Nallapareddy, F.Teng and B.E. Murray. 2004. Influence of origin of isolates, especially endocarditis isolates, and various genes on biofilm formation by Enterococcus faecalis. Infect. Immun. 72: 3658-3663.

Mohamed J.A. and B.E. Murray. 2005. Lack of correlation of gelatinase production and biofilm formation in a large collection of Enterococcus faecalis isolates. J. Clin. Microbiol. 43: 5405-5407.

Nakayama J., Y. Cao, T. Horii, S. Sakuda, A.D. Akkermans, W.M. de Vos and H. Nagasawa. 2001. Gelatinase biosynthesisactivating pheromone: a peptide lactone that mediates a quorum sensing in Enterococcus faecalis. Mol. Microbiol. 41: 145-154.

Nakayama J., S. Chen, N. Oyama, K. Nishiguchi, E.A. Azab, E. Tanaka, R. Kariyama and K. Sonomoto. 2006. Revised model for Enterococcus faecalis fsr quorum-sensing system: the small open reading frame $f s r D$ encodes the gelatinase biosynthesis-activating pheromone propeptide corresponding to staphylococcal AgrD. J. Bacteriol. 188: 8321-8326.

Nakayama J., R. Kariyama and H. Kumon. 2002. Description of a 23.9-kilobase chromosomal deletion containing a region encoding $f_{s} r$ genes which mainly determines the gelatinase-negative phenotype of clinical isolates of Enterococcus faecalis in urine. Appl. Environ. Microbiol. 68: 3152-3155.

Piekarska K., R. Gierczyński, M. Ławrynowicz-Paciorek, M. Kochman and M. Jagielski. 2008. Novel gyrase mutations and characterization of ciprofloxacin-resistant clinical strains of Enterococcus faecalis isolated in Poland. Pol. J. Microbiol. 57: 121-124.

Piekarska K. and M. Jagielski. 2007. Prevalence of virulence-associated genes of Enterococcus faecalis clinical strains isolated from patients and volunteers (in Polish). Med. Dosw. Mikrobiol. 59: 207-216.

Pillai S.K., G. Sakoulas, H.S. Gold, C. Wennersten, G.M. Eliopoulos, R.C. Moellering, Jr. and R.T. Inouye. 2002. Prevalence of the $f s r$ locus in Enterococcus faecalis infections. J. Clin. Microbiol. 40: 2651-2652.

Qin X., K.V. Singh, G.M. Weinstock and B.E. Murray. 2000. Effects of Enterococcus faecalis $f$ s $r$ genes on production of gelatinase and a serine protease and virulence. Infect. Immun. 68: 2579-2586.

Qin X., K.V. Singh, G.M. Weinstock and B.E. Murray. 2001. Characterization of $f_{s} r$, a regulator controlling expression of gelatinase and serine protease in Enterococcus faecalis OG1RF. J. Bacteriol. 183: 3372-3382.

Roberts J.C., K.V. Singh, P.C. Okhuysen and B.E. Murray. 2004. Molecular epidemiology of the $f s r$ locus and of gelatinase production among different subsets of Enterococcus faecalis isolates. J. Clin. Microbiol. 42: 2317-2320.

Rudy M., M. Zientara, T. Bek and G. Martirosian. 2005. Occurrence of Antibiotic Resistant Enterococci in Clinical Specimens from a Pediatric Hospital. Pol. J. Microbiol. 54: 77-80.

Ruiz-Garbajosa P., M.J. Bonten, D.A. Robinson, J. Top, S.R. Nallapareddy, C. Torres, T.M. Coque, R. Canton, F. Baquero, B.E. Murray and others. 2006. Multilocus sequence typing scheme for Enterococcus faecalis reveals hospital-adapted genetic complexes in a background of high rates of recombination. J. Clin. Microbiol. 44: 2220-2228.

Sedgley C.M., A. Molander, S.E. Flannagan, A.C. Nagel, O.K. Appelbe, D.B. Clewell and G. Dahlen. 2005. Virulence, phenotype and genotype characteristics of endodontic Enterococcus spp. Oral Microbiol. Immunol. 20: 10-19.

Sifri C.D., E. Mylonakis, K.V. Singh, X. Qin, D. A. Garsin, B.E. Murray, F.M. Ausubel and S.B. Calderwood. 2002. Virulence effect of Enterococcus faecalis protease genes and the quorum-sensing locus $f s r$ in Caenorhabditis elegans and mice. Infect. Immun. 70: 5647-5650. Su Y. A., M.C. Sulavik, P. He, K.K. Makinen, P.L. Makinen, S. Fiedler, R. Wirth and D.B. Clewell. 1991. Nucleotide sequence 
of the gelatinase gene ( $g e l E)$ from Enterococcus faecalis subsp. liquefaciens. Infect. Immun. 59: 415-420.

Thomas V.C., Y. Hiromasa, N. Harms, L. Thurlow, J. Tomich and L.E. Hancock. 2009. A fratricidal mechanism is responsible for eDNA release and contributes to biofilm development of Enterococcus faecalis. Mol. Microbiol. 72: 1022-1036.

Thomas V.C., L.R. Thurlow, D. Boyle and L.E. Hancock. 2008. Regulation of autolysis-dependent extracellular DNA release by Enterococcus faecalis extracellular proteases influences biofilm development. J. Bacteriol. 190: 5690-5698.

Thurlow L.R., V.C. Thomas, S. Narayanan, S. Olson, S.D. Fleming and L.E. Hancock. 2010. Gelatinase contributes to the pathogenesis of endocarditis caused by Enterococcus faecalis. Infect. Immun. 78: 4936-4943.

Waters C.M., M.H. Antiporta, B.E. Murray and G.M. Dunny. 2003. Role of the Enterococcus faecalis GelE protease in determination of cellular chain length, supernatant pheromone levels, and degradation of fibrin and misfolded surface proteins. J. Bacteriol. 185: 3613-3623.

Werner G., T.M. Coque, A.M. Hammerum, R. Hope, W. Hryniewicz, A. Johnson, I. Klare, K.G. Kristinsson, R. Leclercq, C.H. Lester and others. 2008. Emergence and spread of vancomycin resistance among enterococci in Europe. Euro. Surveill. 13: pii $=19046$. 